\title{
Generalized Abel-Plana Formula as a Renormalization Tool in Quantum Field Theory with Boundaries
}

\author{
Aram Saharian* \\ Department of Physics, Yerevan State University, \\ 375025 Yerevan, Armenia \\ and \\ Departamento de Física-CCEN, Universidade Federal da Paraíba, \\ 58.059-970, Caixa Postal 5.008, João Pessoa, PB, Brazil \\ E-mail: saharyan@server.physdep.r.am
}

\begin{abstract}
We apply the generalized Abel-Plana formula for the investigation of one-loop quantum effects on manifolds with boundaries. This allows to extract from the vacuum expectation values of local physical observables the parts corresponding to the geometry without boundaries and to present the boundary-induced parts in terms of integrals strongly convergent for the points away from the boundaries. As a result, the renormalization procedure for these observables is reduced to the corresponding procedure for the bulks without boundaries.
\end{abstract}

Fifth International Conference on Mathematical Methods in Physics

24 - 28, April 2006

Rio de Janeiro, Brazil

${ }^{*}$ Speaker. 


\section{Introduction}

In many physical problems we need to consider the model on background of manifolds with boundaries on which the dynamical variables satisfy some prescribed boundary conditions. In quantum field theory the imposition of boundary conditions leads to the modification of the spectrum for the zero-point fluctuations and results in the shift in the vacuum expectation values for physical observables. In particular, vacuum forces arise acting on the constraining boundary. This is the familiar Casimir effect (see Refs. [1, 2, 3]). Within the framework of the mode summation method, in calculations of the expectation values for physical observables one often needs to sum over the values of a certain function at integer points, and then subtract the corresponding quantity for unbounded space (usually presented in terms of integrals). Practically, the sum and integral, taken separately, diverge and some physically motivated procedure to handle the finite result, is needed (for a discussion of different methods to evaluate this finite difference see Refs. [4]). For a number of geometries one of the most convenient methods to obtain the renormalized values of the mode-sums is based on the use of the Abel-Plana formula (APF) [5]. In Ref. [6] this formula has been used for the investigation of the expectation values of the energy momentum-tensor for a scalar field on cosmological backgrounds. Further applications to the Casimir effect with corresponding references can be found in [1,2]. The use of the APF allows to extract in a manifestly cutoff independent way the contribution of the unbounded space and present the renormalized values in terms of exponentially converging integrals.

The applications of the APF in its usual form is restricted to the problems where the eigenmodes have simple dependence on quantum numbers and the normal modes are explicitly known. For the case of curved boundaries and for mixed boundary conditions the normal modes are given implicitly as zeroes of the corresponding eigenfunctions or their combinations. To include more general class of problems, in Ref. [7] the APF has been generalized (see also Ref. [8]). The generalized version contains two meromorphic functions and the APF is obtained by specifying one of them (for other generalizations of the APF see $[2,4]$ ). Applying the generalized formula to Bessel functions in Refs. [7, 8] summation formulae are obtained for the series over the zeros of various combinations of these functions (for a review with physical applications see also Ref. [9]). In the present paper we give applications of the generalized Abel-Plana formula to physical problems. The paper is organized as follows. In the next section we briefly outline the generalized Abel-Plana formula and discuss special cases. In section 3 we consider the vacuum polarization by spherical boundaries on background of global monopole spacetime. The application of the generalized AbelPlana formula to the problem of the influence of uniformly accelerated mirrors on the properties of the Fulling-Rindler vacuum is given in section 4. Section 5 describes some other applications.

\section{Generalized Abel-Plana formula}

Let $f(z)$ and $g(z)$ be meromorphic functions for $a \leqslant x \leqslant b$ in the complex plane $z=x+i y$. We denote by $z_{f, k}$ and $z_{g, k}$ the poles of $f(z)$ and $g(z)$ in the region $a<x<b$, respectively, and assume that $\operatorname{Im} z_{f, k} \neq 0$. By using the residue theorem, it can be seen [7] that if the functions $f(z)$ and $g(z)$ 
satisfy the conditions

$$
\lim _{h \rightarrow \infty} \int_{a \pm i h}^{b \pm i h} d z[g(z) \pm f(z)]=0, \lim _{b \rightarrow \infty} \int_{b}^{b \pm i \infty} d z[g(z) \pm f(z)]=0,
$$

and the integral $\int_{a}^{b} d x f(x)$ converges, then

$$
\lim _{b \rightarrow \infty}\left\{\int_{a}^{b} d x f(x)-R[f(z), g(z)]\right\}=\frac{1}{2} \int_{a-i \infty}^{a+i \infty} d z[g(z)+\operatorname{sgn}(\operatorname{Im} z) f(z)],
$$

where

$$
R[f(z), g(z)]=\pi i\left[\sum_{k} \operatorname{Res}_{z=z_{g, k}} g(z)+\sum_{k} \operatorname{Res}_{\operatorname{Im} z_{f, k}>0} f(z)-\sum_{k} \operatorname{Res}_{\operatorname{Im} z_{f, k}<0} f(z)\right] .
$$

We will refer to formula (2.2) as generalized Abel-Plana formula (GAPF) as for $b=n+a, 0<a<$ $1, g(z)=-i f(z) \cot \pi z$, and for analytic functions $f(z)$ from (2.2) follows the APF [5]

$$
\lim _{n \rightarrow \infty}\left[\sum_{1}^{n} f(s)-\int_{a}^{n+a} d x f(x)\right]=\frac{1}{2 i} \int_{a}^{a-i \infty} d z f(z)(\cot \pi z-i)-\frac{1}{2 i} \int_{a}^{a+i \infty} d z f(z)(\cot \pi z+i) .
$$

The useful form of (2.4) may be obtained performing the limit $a \rightarrow 0$. By taking into account that the point $z=0$ is a pole for the integrands and therefore has to be avoided by arcs of the small circle $C_{\rho}$ on the right and performing $\rho \rightarrow 0$, one obtains

$$
\sum_{n=0}^{\infty} f(n)=\int_{0}^{\infty} d x f(x)+\frac{1}{2} f(0)+i \int_{0}^{\infty} d x \frac{f(i x)-f(-i x)}{e^{2 \pi x}-1} .
$$

Note that now conditions (2.1) are satisfied if $\lim _{y \rightarrow \infty} e^{-2 \pi|y|}|f(x+i y)|=0$, uniformly in any finite interval of $x$. Formula (2.5) is the most frequently used form of the APF in physical applications. Another useful form [1] (in particular, for fermionic field calculations) to sum over the values of an analytic function at half of an odd integer points can be obtained from (2.5).

Formula (2.2) contains two meromorphic functions and is too general. To obtain more specific consequences we have to specify one of them. In applications we often need to sum over the values of a certain function at the points being the zeros of the other function. In order to obtain the summation formula for this type of series, as a function $g(z)$ we take the function for which these zeros are poles. Then the first term in the square brackets of Eq. (2.3) will give the corresponding series. This choice should be made in a way to meet conditions (2.1). We will illustrate this on specific physical examples of quantum field theory with boundaries.

\section{Vacuum densities for spherical boundaries}

Consider a real scalar field $\varphi$ with curvature coupling parameter $\zeta$ on a $(D+1)$-dimensional spacetime region $M$ with static boundary $\partial M$. The corresponding field equation has the form

$$
\left(\nabla_{i} \nabla^{i}+m^{2}+\zeta R\right) \varphi=0
$$

where $R$ is the scalar curvature for the background spacetime, $m$ is the mass for the field quanta, $\nabla_{i}$ is the covariant derivative operator. We assume that on the boundary the field satisfies the Robin boundary condition

$$
\left(\tilde{A}+\tilde{B} n^{i} \nabla_{i}\right) \varphi(x)=0, x \in \partial M
$$


with constant coefficients $\tilde{A}, \tilde{B}$ and $n^{i}$ being the inward-pointing normal to the boundary. The vacuum expectation values (VEVs) for physical quantities bilinear in the field operator, can be found evaluating one of two-point functions. Here we will consider the Wightman function, as it also determines the response of particle detectors in a given state of motion. This function can be determined from the mode-sum formula

$$
W\left(x, x^{\prime}\right)=\left\langle 0\left|\varphi(x) \varphi\left(x^{\prime}\right)\right| 0\right\rangle=\sum_{\sigma} \varphi_{\sigma}(x) \varphi_{\sigma}^{*}\left(x^{\prime}\right),
$$

where $\left\{\varphi_{\sigma}(x), \varphi_{\sigma}^{*}\left(x^{\prime}\right)\right\}$ is a complete orthonormal set of positive and negative frequency solutions to the field equation, specified by quantum numbers $\sigma$ and satisfying boundary condition (3.2).

\subsection{Vacuum inside a spherical shell in the global monopole spacetime}

As the first example we consider the region inside a spherical shell with radius $a$ in the global monopole spacetime. In the hyperspherical polar coordinates $(r, \vartheta, \phi) \equiv\left(r, \theta_{1}, \theta_{2}, \ldots \theta_{n}, \phi\right), n=$ $D-2$, the corresponding line element has the form

$$
d s^{2}=d t^{2}-d r^{2}-\alpha^{2} r^{2} d \Omega_{D}^{2}
$$

where $d \Omega_{D}^{2}$ is the line element on the surface of unit sphere in $D$-dimensional Euclidean space, the parameter $\alpha$ is smaller than unity and is related to the symmetry breaking energy scale in the theory. The solid angle corresponding to Eq. (3.4) is $\alpha^{2} S_{D}$, with $S_{D}=2 \pi^{D / 2} / \Gamma(D / 2)$ being the total area of the surface of the unit sphere in $D$-dimensional Euclidean space.

For the region inside the sphere the complete set of solutions to Eq. (3.1) is specified by the set of quantum numbers $\sigma=\left(\lambda, m_{k}\right)$, where $m_{k}=\left(m_{0} \equiv l, m_{1}, \ldots, m_{n}\right)$ and $m_{1}, m_{2}, \ldots, m_{n}$ are integers such that $0 \leqslant m_{n-1} \leqslant m_{n-2} \leqslant \cdots \leqslant m_{1} \leq l,-m_{n-1} \leqslant m_{n} \leqslant m_{n-1}$. The corresponding eigenfunctions have the form

$$
\varphi_{\sigma}(x)=\beta_{\sigma} r^{-n / 2} J_{v_{l}}(\lambda r) Y\left(m_{k} ; \vartheta, \phi\right) e^{-i \omega t}, \omega=\sqrt{\lambda^{2}+m^{2}}, l=0,1,2, \ldots,
$$

where $J_{v_{l}}(z)$ is the Bessel function of the order

$$
v_{l}=\frac{1}{\alpha}\left[\left(l+\frac{n}{2}\right)^{2}+\left(1-\alpha^{2}\right) n\left((n+1) \zeta-\frac{n}{4}\right)\right]^{1 / 2},
$$

and $Y\left(m_{k} ; \vartheta, \phi\right)$ is the hyperspherical harmonic of degree $l$. The coefficients $\beta_{\sigma}$ can be found from the normalization condition and is equal to

$$
\beta_{\sigma}^{2}=\frac{\lambda T_{v_{l}}(\lambda a)}{N\left(m_{k}\right) \omega a \alpha^{D-1}}, T_{v}(z) \equiv \frac{z}{\left(z^{2}-v^{2}\right) J_{v}^{2}(z)+z^{2} J_{v}^{\prime 2}(z)} .
$$

From boundary condition (3.2) it follows that the possible values for $\lambda$ are solutions to the equation

$$
A J_{v_{l}}(z)+B z J_{v_{l}}^{\prime}(z)=0, \quad z=\lambda a, \quad A=\tilde{A}-n B / 2, \quad B=-\tilde{B} / a .
$$

It is well known that for real $A, B$, and $v_{l}>-1$ all roots of this equation are simple and real, except the case $A / B<-v_{l}$ when there are two purely imaginary zeros. In the following we will assume 
values of $A / B$ for which all roots are real, $A / B \geq-v_{l}$. Let $\lambda_{v_{l}, k}, k=1,2, \ldots$, be the positive zeros of the function $A J_{v_{l}}(z)+B z J_{v_{l}}^{\prime}(z)$, arranged in ascending order. The corresponding eigenfrequencies $\omega=\omega_{v_{l}, k}$ are related to these zeros by the formula $\omega_{v_{l}, k}=\sqrt{\lambda_{v_{l}, k}^{2} / a^{2}+m^{2}}$. Substituting Eq. (3.5) into Eq. (3.3) and using the addition formula for the spherical harmonics, one obtains

$$
\begin{aligned}
W\left(x, x^{\prime}\right)= & \frac{\left(r r^{\prime}\right)^{-n / 2}}{n a S_{D} \alpha^{D-1}} \sum_{l=0}^{\infty}(2 l+n) C_{l}^{n / 2}(\cos \theta) \\
& \times \sum_{k=1}^{\infty} \frac{\lambda_{v_{l}, k} T_{v_{l}}\left(\lambda_{v_{l}, k}\right)}{\sqrt{\lambda_{v_{l}, k}^{2}+m^{2} a^{2}}} J_{v_{l}}\left(\lambda_{v_{l}, k} r / a\right) J_{v_{l}}\left(\lambda_{v_{l}, k} r^{\prime} / a\right) e^{i \omega_{v_{l}, k}\left(t^{\prime}-t\right)},
\end{aligned}
$$

where $C_{p}^{q}(x)$ is the Gegenbauer polynomial of degree $p$ and order $q$, and $\theta$ is the angle between the directions $(\vartheta, \phi)$ and $\left(\vartheta^{\prime}, \phi^{\prime}\right)$. As the normal modes $\lambda_{v_{l}, k}$ are not explicitly known, the Wightman function in the form (3.9) is not convenient for the evaluation of the VEVs for the observables bilinear in the field. In addition, the terms with large values $k$ are highly oscillatory. In order to obtain summation formula for the series over $k$, as a function $g(z)$ in the GAPF we choose $g(z)=i \bar{Y}_{v}(z) f(z) / \bar{J}_{v}(z)$, where $Y_{v}(z)$ is the Neumann function and for a given function $F(z)$ we use the barred notation

$$
\bar{F}(z) \equiv A F(z)+B z F^{\prime}(z)
$$

The conditions (2.1) are satisfied if the function $f(z)$ is restricted by the constraint

$$
|f(z)|<\varepsilon(x) e^{c|y|}, z=x+i y, \quad|z| \rightarrow \infty,
$$

where $c<2$ and $\varepsilon(x) \rightarrow 0$ for $x \rightarrow \infty$. Assuming that the function $f(z)$ is analytic in the right halfplane, for (2.3) one finds $R[f(z), g(z)]=2 \sum_{k} T_{v}\left(\lambda_{v, k}\right) f\left(\lambda_{v, k}\right)$. Substituting this expression into (2.2) and taking the limit $a \rightarrow 0$, it can be seen that the following summation formula takes place

$$
\begin{aligned}
\sum_{k=1}^{\infty} T_{v}\left(\lambda_{v, k}\right) f\left(\lambda_{v, k}\right)= & \frac{1}{2} \int_{0}^{\infty} d x f(x)+\frac{\pi}{4} \operatorname{Res} f(z) \frac{\bar{Y}_{v}(z)}{\bar{J}_{v}(z)} \\
& -\frac{1}{2 \pi} \int_{0}^{\infty} d x \frac{\bar{K}_{v}(x)}{\bar{I}_{v}(x)}\left[e^{-v \pi i} f(i x)+e^{v \pi i} f(-i x)\right],
\end{aligned}
$$

where we have introduced the modified Bessel functions $I_{v}(x)$ and $K_{v}(x)$. By taking in this formula $v=1 / 2, A=1, B=0$, as a special case we receive the APF in the form (2.5). Formula (3.12) can be generalized in the case of the existence of purely imaginary zeros for the function $\bar{J}_{v}(z)$ by adding the corresponding residue term and taking the principal value of the integral on the right (see Ref. [9]).

For the summation over $k$ in Eq. (3.9) we apply formula (3.12). The corresponding conditions are satisfied if $r+r^{\prime}+\left|t-t^{\prime}\right|<2 a$. In particular, the latter constraint takes place in the coincidence limit for the points away from the boundary. Now the Wightman function is presented in the form

$$
W\left(x, x^{\prime}\right)=W_{\mathrm{m}}\left(x, x^{\prime}\right)+\left\langle\varphi(x) \varphi\left(x^{\prime}\right)\right\rangle_{b}
$$

where the term

$$
W_{\mathrm{m}}\left(x, x^{\prime}\right)=\frac{\alpha^{1-D}}{2 n S_{D}} \sum_{l=0}^{\infty} \frac{2 l+n}{\left(r r^{\prime}\right)^{n / 2}} C_{l}^{n / 2}(\cos \theta) \int_{0}^{\infty} d z \frac{z e^{i \sqrt{z^{2}+m^{2}}\left(t^{\prime}-t\right)}}{\sqrt{z^{2}+m^{2}}} J_{v_{l}}(z r) J_{v_{l}}\left(z r^{\prime}\right),
$$


comes from the first integral on the right of Eq. (3.12) and

$$
\begin{aligned}
\left\langle\varphi(x) \varphi\left(x^{\prime}\right)\right\rangle_{b}= & -\frac{\alpha^{1-D}}{\pi n S_{D}} \sum_{l=0}^{\infty} \frac{2 l+n}{\left(r r^{\prime}\right)^{n / 2}} C_{l}^{n / 2}(\cos \theta) \int_{m}^{\infty} d z z \frac{\bar{K}_{v_{l}}(a z)}{\bar{I}_{v_{l}}(a z)} \\
& \times \frac{I_{v_{l}}(z r) I_{v_{l}}\left(z r^{\prime}\right)}{\sqrt{z^{2}-m^{2}}} \cosh \left[\sqrt{z^{2}-m^{2}}\left(t^{\prime}-t\right)\right] .
\end{aligned}
$$

The part (3.14) in the Wightman function does not depend on $a$, whereas the contribution of the term (3.15) tends to zero as $a \rightarrow \infty$. It follows from here that expression (3.14) is the Wightman function for the unbounded global monopole spacetime. This can be seen also by explicit evaluation of the mode-sum using the eigenfunctions for the global monopole spacetime without boundaries.

Having the Wightman function we can evaluate the VEVs for the field square and the energymomentum tensor taking the following coincidence limits:

$$
\begin{aligned}
& \left\langle 0\left|\varphi^{2}\right| 0\right\rangle=\lim _{x^{\prime} \rightarrow x} W\left(x, x^{\prime}\right), \\
& \left\langle 0\left|T_{i k}\right| 0\right\rangle=\lim _{x^{\prime} \rightarrow x} \partial_{i} \partial_{k}^{\prime} W\left(x, x^{\prime}\right)+\left[\left(\zeta-\frac{1}{4}\right) g_{i k} \nabla_{l} \nabla^{l}-\zeta \nabla_{i} \nabla_{k}-\zeta R_{i k}\right]\left\langle 0\left|\varphi^{2}\right| 0\right\rangle .
\end{aligned}
$$

Similar to the Wightman function, these VEVs are presented as sums of the boundary-free and boundary-induced parts and are investigated in Ref. [10]. Hence, the application of the GAPF allowed to extract from the VEVs the parts corresponding to the background without boundaries. For points away from the boundaries, the boundary-induced parts are finite and the renormalization procedure is needed for the boundary-free part only. This can be done by using the standard methods of quantum field theory without boundaries. In addition, the boundary-induced parts are presented in terms of exponentially convergent integrals convenient for numerical calculations.

For $\alpha=1$ the bulk corresponds to the $(D+1)$-dimensional Minkowskian spacetime and one has $v_{l}=l+n / 2$. In this case the vacuum densities for a scalar field with Robin boundary conditions on spherical boundaries are investigated in Ref. [11]. In the case of the electromagnetic field on the $D=3$ Minkowski bulk we have two types of modes. For the first one (TE modes) the eigenmodes are zeroes of the function $J_{l+1 / 2}(z)$, and for the second one the eigenmodes are determined by the zeroes of the function $\bar{J}_{l+1 / 2}(z)$ with $A / B=1 / 2$. On the base of the GAPF the corresponding VEVs for the energy-momentum tensor are investigated in Ref. [12].

\subsection{Region between two spheres}

In this section we are interested in the VEVs of the field bilinear products on background of the geometry described by Eq. (3.4), assuming that the field satisfies the Robin boundary condition (3.2) on two spheres with radii $a$ and $b, a<b$, concentric with the monopole. We will consider the general case when the coefficients in the boundary conditions for the inner and outer spheres are different and will denote them by $\tilde{A}_{j}$ and $\tilde{B}_{j}$ with $j=a, b$. The corresponding eigenfunctions are given by the formula which is obtained from (3.5) by the replacement of the radial part:

$$
J_{v_{l}}(\lambda r) \rightarrow g_{v_{l}}(\lambda a, \lambda r) \equiv J_{v_{l}}(\lambda r) \bar{Y}_{v_{l}}^{(a)}(\lambda a)-\bar{J}_{v_{l}}^{(a)}(\lambda a) Y_{v_{l}}(\lambda r)
$$

and the barred functions are defined in accordance with

$$
\bar{F}^{(j)}(z) \equiv A_{j} F(z)+B_{j} z F^{\prime}(z), \quad A_{\alpha}=\tilde{A}_{j}-B_{j} n / 2, \quad B_{j}=n^{(j)} \tilde{B}_{j} / j, j=a, b,
$$


where $n^{(a)}=-1$ and $n^{(b)}=1$. From the normalization condition, for the coefficient in the eigenfunctions one finds

$$
\beta_{\sigma}^{2}=\frac{\pi^{2} \lambda T_{v_{l}}^{a b}(b / a, \lambda a)}{4 N\left(m_{k}\right) \omega a \alpha^{D-1}}
$$

where we use the notation

$$
T_{v}^{a b}(\eta, z)=z\left\{\frac{\bar{J}_{v}^{(a) 2}(z)}{\bar{J}_{v}^{(b) 2}(\eta z)}\left[A_{b}^{2}+B_{b}^{2}\left(\eta^{2} z^{2}-v^{2}\right)\right]-A_{a}^{2}-B_{a}^{2}\left(z^{2}-v^{2}\right)\right\}^{-1}, \quad \eta=\frac{b}{a} .
$$

The functions chosen in the form (3.18) satisfy the boundary condition on the sphere $r=a$. From the boundary condition on $r=b$ one obtains that the corresponding eigenvalues for the quantum number $\lambda$ are solutions to the equation

$$
C_{v_{l}}^{a b}(b / a, \lambda a) \equiv \bar{J}_{v_{l}}^{(a)}(\lambda a) \bar{Y}_{v_{l}}^{(b)}(\lambda b)-\bar{J}_{v_{l}}^{(b)}(\lambda b) \bar{Y}_{v_{l}}^{(a)}(\lambda a)=0
$$

Below the roots to this equation will be denoted by $\lambda a=\gamma_{v_{l}, k}, k=1,2, \ldots$

Substituting the eigenfunctions into the mode-sum formula, one finds

$$
W\left(x, x^{\prime}\right)=\frac{\pi^{2}\left(r r^{\prime}\right)^{-n / 2}}{4 n a S_{D} \alpha^{D-1}} \sum_{l=0}^{\infty}(2 l+n) C_{l}^{n / 2}(\cos \theta) \sum_{k=1}^{\infty} h\left(\gamma_{v_{l}, k}\right) T_{v_{l}}^{a b}\left(b / a, \gamma_{v_{l}, k}\right),
$$

with the function

$$
h(z)=\frac{z e^{i \sqrt{z^{2} / a^{2}+m^{2}}\left(t^{\prime}-t\right)}}{\sqrt{z^{2}+m^{2} a^{2}}} g_{v_{l}}(z, z r / a) g_{v_{l}}\left(z, z r^{\prime} / a\right) .
$$

To obtain a summation formula for the series over zeros of the function $C_{v}^{a b}(\eta, z)$, in the GAPF as functions $g(z)$ and $f(z)$ we take

$$
g(z)=\frac{1}{2 i}\left[\frac{\bar{H}_{v}^{(1 b)}(\eta z)}{\bar{H}_{v}^{(1 a)}(z)}+\frac{\bar{H}_{v}^{(2 b)}(\eta z)}{\bar{H}_{v}^{(2 a)}(z)}\right] \frac{F(z)}{C_{v}^{a b}(\eta, z)}, \quad f(z)=\frac{F(z)}{\bar{H}_{v}^{(1 a)}(z) \bar{H}_{v}^{(2 a)}(z)},
$$

where $F(z)$ is an analytic function in the right half-plane, $H_{v}^{(1,2)}(z)$ are the Hankel functions, and the notations $\bar{F}^{(j)}, j=a, b$ are introduced in accordance with (3.19). For the combinations entering in the right hand side of the GAPF we obtain

$$
g(z)-(-1)^{q} f(z)=-i \frac{\bar{H}_{v}^{(q a)}(\lambda z)}{\bar{H}_{v}^{(q a)}(z)} \frac{F(z)}{C_{v}^{a b}(\eta, z)}, \quad q=1,2,
$$

and for the residue term one has

$$
\operatorname{Res}_{z=\gamma_{v, k}} g(z)=\frac{\pi}{2 i} T_{v}^{a b}\left(\eta, \gamma_{v, k}\right) F\left(\gamma_{v, k}\right)
$$

The conditions for the GAPF, written in terms of the function $F(z)$, are as follows

$$
|F(z)|<\varepsilon_{1}(x) e^{c_{1}|y|}, \quad|z| \rightarrow \infty, \quad z=x+i y,
$$


where $c_{1}<2(\eta-1), x^{\delta_{B_{a} 0}+\delta_{B_{b} 0}-1} \varepsilon_{1}(x) \rightarrow 0$ for $x \rightarrow+\infty$. From Eq. (2.2) we obtain the following summation formula

$$
\begin{aligned}
\sum_{k=1}^{\infty} F\left(\gamma_{v, k}\right) T_{v}^{a b}\left(\eta, \gamma_{v, k}\right)= & \frac{2}{\pi^{2}} \int_{0}^{\infty} \frac{F(x) d x}{\bar{J}_{v}^{(a) 2}(x)+\bar{Y}_{v}^{(a) 2}(x)}-\frac{1}{\pi} \operatorname{Res}\left[\frac{F(z) \bar{H}_{v}^{(1 b)}(\eta z)}{C_{v}^{a b}(\eta, z) \bar{H}_{v}^{(1 a)}(z)}\right] \\
& -\frac{1}{2 \pi} \int_{0}^{\infty} d x \Omega_{a v}(x, \eta x)[F(i x)+F(-i x)] .
\end{aligned}
$$

In Eq. (3.29) we have introduced the notation

$$
\Omega_{a v}(x, \eta x)=\frac{\bar{K}_{v}^{(b)}(\eta x) / \bar{K}_{v}^{(a)}(x)}{\bar{K}_{v}^{(a)}(x) \bar{I}_{v}^{(b)}(\eta x)-\bar{K}_{v}^{(b)}(\eta x) \bar{I}_{v}^{(a)}(x)} .
$$

Note that (3.29) may be generalized for the functions $F(z)$ having poles and in the case of the existence of purely imaginary zeros for $C_{v}^{a b}(\eta, z)$ [9].

Now, to sum the series over $k$ in Eq. (3.23), we take in (3.29) $F(z)=h(z)$. The corresponding conditions are satisfied if $r+r^{\prime}+\left|t-t^{\prime}\right|<2 b$. In particular, this is the case in the coincidence limit for the region between two spheres. As a result, for the Wightman function one obtains

$$
\begin{aligned}
W\left(x, x^{\prime}\right)= & \frac{\alpha^{1-D}}{2 n a S_{D}} \sum_{l=0}^{\infty} \frac{2 l+n}{\left(r r^{\prime}\right)^{n / 2}} C_{l}^{n / 2}(\cos \theta)\left\{\int_{0}^{\infty} \frac{h(z) d z}{\bar{J}_{v_{l}}^{(a) 2}(z)+\bar{Y}_{v_{l}}^{(a) 2}(z)}-\frac{2}{\pi} \int_{m a}^{\infty} d z z\right. \\
& \left.\times \frac{\Omega_{a v}(z, \eta z)}{\sqrt{z^{2}-a^{2} m^{2}}} G_{v_{l}}^{(a)}(z, z r / a) G_{v_{l}}^{(a)}\left(z, z r^{\prime} / a\right) \cosh \left[\sqrt{z^{2} / a^{2}-m^{2}}\left(t^{\prime}-t\right)\right]\right\}
\end{aligned}
$$

where we have introduced the notation

$$
G_{v}^{(j)}(z, y)=I_{v}(y) \bar{K}_{v}^{(j)}(z)-\bar{I}_{v}^{(j)}(z) K_{v}(y), j=a, b .
$$

In the limit $b \rightarrow \infty$ the second integral on the right of (3.31) tends to zero, whereas the first one does not depend on $b$. It follows from here that the term with the first integral in the figure braces corresponds to the Wightman function for the region outside a single sphere with radius $a$ on background of the global monopole geometry. The latter we will denote by $W^{(a)}\left(x, x^{\prime}\right)$. To extract from this function the part induced by the presence of the sphere we use the identity

$$
\frac{g_{v}(z, z r / a) g_{v}\left(z, z r^{\prime} / a\right)}{\bar{J}_{v}^{2}(z)+\bar{Y}_{v}^{2}(z)}=J_{v}(z r / a) J_{v}\left(z r^{\prime} / a\right)-\frac{1}{2} \sum_{s=1}^{2} \frac{\bar{J}_{v}(z)}{\bar{H}_{v}^{(s)}(z)} H_{v}^{(s)}(z r / a) H_{v}^{(s)}\left(z r^{\prime} / a\right) .
$$

The contribution from the first term on the right of this relation gives the Wightman function $W_{\mathrm{m}}\left(x, x^{\prime}\right)$ for the geometry without boundaries. In the part coming from the second term we rotate the contour for the integration over $z$ by the angle $\pi / 2$ for $s=1$ and by the angle $-\pi / 2$ for $s=2$. Introducing the modified Bessel functions, one finds

$$
\begin{aligned}
W^{(a)}\left(x, x^{\prime}\right)= & W_{\mathrm{m}}\left(x, x^{\prime}\right)-\frac{\alpha^{1-D}}{\pi n S_{D}} \sum_{l=0}^{\infty} \frac{2 l+n}{\left(r r^{\prime}\right)^{n / 2}} C_{l}^{n / 2}(\cos \theta) \int_{m}^{\infty} d z z \\
& \times \frac{\bar{I}_{V_{l}}(a z)}{\bar{K}_{v_{l}}(a z)} \frac{K_{v_{l}}(z r) K_{V_{l}}\left(z r^{\prime}\right)}{\sqrt{z^{2}-m^{2}}} \cosh \left[\sqrt{z^{2}-m^{2}}\left(t^{\prime}-t\right)\right] .
\end{aligned}
$$


As a result the Wightman function in the region between two spheres is presented in the form

$$
\begin{aligned}
W\left(x, x^{\prime}\right)= & W^{(a)}\left(x, x^{\prime}\right)-\frac{\alpha^{1-D}}{\pi n S_{D}} \sum_{l=0}^{\infty} \frac{2 l+n}{\left(r r^{\prime}\right)^{n / 2}} C_{l}^{n / 2}(\cos \theta) \int_{m}^{\infty} d z z \frac{\Omega_{a v_{l}}(a z, b z)}{\sqrt{z^{2}-m^{2}}} \\
& \times G_{v_{l}}^{(a)}(a z, r z) G_{v_{l}}^{(a)}\left(a z, r^{\prime} z\right) \cosh \left[\sqrt{z^{2}-m^{2}}\left(t^{\prime}-t\right)\right] .
\end{aligned}
$$

In the coincidence limit, $x^{\prime}=x$, the second summand on the right hand side of (3.35) will give a finite result for $a \leqslant r<b$, and is divergent on the boundary $r=b$. Hence, in the problem with two boundaries, the GAPF allowed to extract the part corresponding to the geometry with a single boundary and to present the part induced by the second boundary in terms of exponentially convergent integrals. The VEVs for the field square and the energy-momentum tensor in the region between two spheres are obtained from the Wightman function on the base of formulae (3.16), (3.17) and are investigated in Ref. [13].

We have considered the case of a scalar field. The VEVs for the energy-momentum tensor of a fermionic field $\psi$ with the mass $m$ on background of the global monopole have been discussed in Ref. [14] for the case of a single spherical boundary and in Ref. [15] for the geometry of two spherical boundaries assuming that the field satisfies bag boundary condition $\left(1+i \gamma^{l} n_{l}\right) \psi=0$ on bounding surfaces. For the region inside a spherical shell with radius $a$ the corresponding eigenmodes are the zeroes of the function $\tilde{J}_{v_{\sigma}}^{(a)}(z)$, where for a given function $F(z)$ we use the notation

$$
\tilde{F}^{(w)}(z) \equiv z F^{\prime}(z)+\left[n^{(w)}\left(m w-\sqrt{z^{2}+m^{2} w^{2}}\right)-(-1)^{\sigma} v_{\sigma}\right] F(z),
$$

with $n^{(a)}=1, v_{\sigma}=(j+1 / 2) / \alpha-(-1)^{\sigma} / 2, j$ is the total angular momentum, and $\sigma=0,1$ correspond to two types of eigenfunctions with different parities. By using the GAPF, a summation formula for the series over zeroes of the function $\tilde{J}_{v_{\sigma}}^{(a)}(z)$ is derived in Ref. [14]. In the region between two concentric spherical boundaries with radii $a$ and $b, a>b$, on the global monopole bulk, the eigenmodes of the fermionic field are the zeroes of the function $\tilde{J}_{v_{\sigma}}^{(b)}(z) \tilde{Y}_{v_{\sigma}}^{(a)}(z a / b)-$ $\tilde{Y}_{v_{\sigma}}^{(b)}(z) \tilde{J}_{v_{\sigma}}^{(a)}(z a / b)$ with $n^{(b)}=-1$. A summation formula for the corresponding series is obtained in Ref. [15] and has been applied to the investigation of the corresponding VEVs. The properties of the electromagnetic vacuum in the region between two concentric spheres in the Minkowski bulk are studied in Ref. [16] by using the GAPF.

\section{Vacuum polarization by uniformly accelerated mirrors}

It is well known that the uniqueness of the vacuum state is lost when we work within the framework of quantum field theory in a general curved spacetime or in non-inertial frames. For instance, the vacuum state for a uniformly accelerated observer, the Fulling-Rindler vacuum, turns out to be inequivalent to that for an inertial observer. An interesting topic in the investigations of the Casimir effect is the dependence of the vacuum characteristics on the type of the vacuum. In this section we will consider the application of the GAPF for the investigation of the scalar vacuum polarization brought about by the presence of infinite plane boundaries moving by uniform proper acceleration through the Fulling-Rindler vacuum. 


\subsection{Wightman function for a single plate}

Consider a massive scalar field with general curvature coupling and satisfying Robin boundary condition on an infinite plane moving with uniform proper acceleration. In the accelerated frame it is convenient to use the Rinlder coordinates with the line element

$$
d s^{2}=\xi^{2} d \tau^{2}-d \xi^{2}-d \mathbf{x}^{2} .
$$

A world-line defined by $\xi, \mathbf{x}=$ const describes an observer with constant proper acceleration $\xi^{-1}$. We will assume that the plate is located at $\xi=a$ and will consider the region on the right from the boundary, $\xi \geqslant a$, where $a^{-1}$ is the proper acceleration of the plate. In the Rindler coordinates, boundary condition (3.2) takes the form

$$
(\tilde{A}+\tilde{B} \partial / \partial \xi) \varphi(x)=0, \quad \xi=a .
$$

For $\xi \geqslant a$ a complete set of solutions that are of positive frequency with respect to $\partial / \partial \tau$ and bounded as $\xi \rightarrow \infty$ is

$$
\varphi_{\sigma}(x)=C_{\sigma} K_{i \omega}(\lambda \xi) e^{i \mathbf{k x}-i \omega \tau}, \quad \sigma=(\omega, \mathbf{k}), \lambda=\sqrt{k^{2}+m^{2}} .
$$

From boundary condition (4.2) we find that the possible values for $\omega$ have to be zeros of the function $\bar{K}_{i \omega}(\lambda a)$, where the barred notation is defined by (3.10) with the coefficients $A=\tilde{A}, B=$ $\tilde{B} / a$. We will denote these zeros by $\omega=\omega_{n}=\omega_{n}(k), n=1,2, \ldots$ arranged in ascending order, $\omega_{n}<\omega_{n+1}$. The coefficient $C_{\sigma}$ in (4.3) is determined by the normalization condition:

$$
C_{\sigma}^{2}=\frac{1}{(2 \pi)^{D-1}} \frac{\bar{I}_{i \omega_{n}}(\lambda a)}{\left.\frac{\partial}{\partial \omega} \bar{K}_{i \omega}(\lambda a)\right|_{\omega=\omega_{n}}} .
$$

Substituting the eigenfunctions (4.3) into the mode-sum formula (3.3), we obtain

$$
W\left(x, x^{\prime}\right)=\left.\int \frac{d \mathbf{k}}{(2 \pi)^{D-1}} e^{i \mathbf{k}\left(\mathbf{x}-\mathbf{x}^{\prime}\right)} \sum_{n=1}^{\infty} \frac{\bar{I}_{i \omega}(\lambda a)}{\frac{\partial}{\partial \omega} \bar{K}_{i \omega}(\lambda a)} K_{i \omega}(\lambda \xi) K_{i \omega}\left(\lambda \xi^{\prime}\right) e^{-i \omega\left(\tau-\tau^{\prime}\right)}\right|_{\omega=\omega_{n}} .
$$

A summation formula for the series over zeros $\omega_{n}$ can be obtained from formula (2.2) taking

$$
f(z)=\frac{2 i}{\pi} F(z) \sinh \pi z, \quad g(z)=\frac{\bar{I}_{i z}(\eta)+\bar{I}_{-i z}(\eta)}{\bar{K}_{i z}(\eta)} F(z),
$$

with a function $F(z)$ analytic in the right half-plane. By using the asymptotic formulae for the modified Bessel functions for large values of the index, the conditions for the GAPF can be written in terms of the function $F(z)$ as follows:

$$
|F(z)|<\varepsilon(|z|) e^{-\pi x}(|z| / \eta)^{2|y|}, \quad z=x+i y, \quad x>0, \quad|z| \rightarrow \infty,
$$

where $|z| \varepsilon(|z|) \rightarrow 0$ for $|z| \rightarrow \infty$. From the GAPF we obtain the summation formula

$$
\left.\sum_{n=1}^{\infty} \frac{\bar{I}_{i z}(\eta) F(z)}{\partial \bar{K}_{i z}(\eta) / \partial z}\right|_{z=\omega_{n}}=\frac{1}{\pi^{2}} \int_{0}^{\infty} d x F(x) \sinh \pi x-\frac{F_{0} \bar{I}_{0}(\eta)}{2 \bar{K}_{0}(\eta)}-\int_{0}^{\infty} d x \frac{\bar{I}_{x}(\eta)}{\bar{K}_{x}(\eta)} \frac{F(i x)+F(-i x)}{2 \pi}
$$

where $F_{0}=\lim _{z \rightarrow 0} z F(z)$. 
Now for the summation over $n$ in formula (4.5) we choose $F(z)=K_{i z}(\lambda \xi) K_{i z}\left(\lambda \xi^{\prime}\right) e^{-i z\left(\tau-\tau^{\prime}\right)}$. It can be seen that condition (4.7) is satisfied if $a^{2} e^{\left|\tau-\tau^{\prime}\right|}<\left|\xi \xi^{\prime}\right|$. Note that this is the case in the coincidence limit $\tau=\tau^{\prime}$ for the points in the region under consideration, $\xi_{,} \xi^{\prime}>a$. The contribution coming from the first integral term on the right of formula (4.8) corresponds to the Wightman function for the Fulling-Rindler vacuum without boundaries:

$$
W_{\mathrm{R}}\left(x, x^{\prime}\right)=\frac{1}{\pi^{2}} \int d \mathbf{k} \frac{e^{i \mathbf{k}\left(\mathbf{x}-\mathbf{x}^{\prime}\right)}}{(2 \pi)^{D-1}} \int_{0}^{\infty} d \omega \sinh (\pi \omega) e^{-i \omega\left(\tau-\tau^{\prime}\right)} K_{i \omega}(\lambda \xi) K_{i \omega}\left(\lambda \xi^{\prime}\right)
$$

As a result, the Wightman function is presented in the form

$$
W\left(x, x^{\prime}\right)=W_{\mathrm{R}}\left(x, x^{\prime}\right)+\left\langle\varphi(x) \varphi\left(x^{\prime}\right)\right\rangle_{\mathrm{b}},
$$

where the second term on the right is induced by the presence of the plate:

$$
\left\langle\varphi(x) \varphi\left(x^{\prime}\right)\right\rangle_{\mathrm{b}}=-\frac{1}{\pi} \int d \mathbf{k} \frac{e^{i \mathbf{k}\left(\mathbf{x}-\mathbf{x}^{\prime}\right)}}{(2 \pi)^{D-1}} \int_{0}^{\infty} d \omega \frac{\bar{I}_{\omega}(\lambda a)}{\bar{K}_{\omega}(\lambda a)} K_{\omega}(\lambda \xi) K_{\omega}\left(\lambda \xi^{\prime}\right) \cosh \left[\omega\left(\tau-\tau^{\prime}\right)\right],
$$

and is finite in the coincidence limit for $\xi>a$. The divergences are contained in the first term corresponding to the Fulling-Rindler vacuum without boundaries. The VEVs of the field square and the energy-momentum tensor can be found by making use of the formulae for the Wightman function and relations (3.16) and (3.17). The corresponding results can be found in Ref. [17].

\subsection{Wightman function in the region between two plates}

Now we consider the vacuum in the region between two plates situated in the right Rindler wedge and having the coordinates $\xi=a$ and $\xi=b, b>a$. On the surfaces of the plates the scalar field satisfies Robin boundary conditions (3.2), in general, with different coefficients for separate plates. In terms of the Rindler coordinate $\xi$ these conditions are written in the form $\left(\tilde{A}_{j}+n^{(j)} \tilde{B}_{j} \partial / \partial \xi\right) \varphi=0$, for $\xi=j=a, b$. In the region between the plates, the eigenfunctions satisfying the boundary condition on the plate $\xi=b$ have the form

$$
\varphi_{\sigma}(x)=C_{\sigma} G_{i \omega}^{(b)}(\lambda b, \lambda \xi) e^{i \mathbf{k x}-i \omega \tau}
$$

where the function $G_{v}^{(j)}(u, v)$ is defined by formula (3.32). From the boundary condition on the plate $\xi=a$ we find that the possible values for $\omega$ are roots to the equation

$$
G_{i \omega}^{a b}(\lambda a, \lambda b)=\bar{I}_{i \omega}^{(b)}(\lambda b) \bar{K}_{i \omega}^{(a)}(\lambda a)-\bar{K}_{i \omega}^{(b)}(\lambda b) \bar{I}_{i \omega}^{(a)}(\lambda a)=0
$$

and the barred notations are defined by formula (3.19) with $A_{j}=\tilde{A}_{j}, B_{j}=n^{(j)} \tilde{B}_{j} / j$. For a fixed $\lambda$, the equation (4.13) has an infinite set of real solutions with respect to $\omega$. We will denote them by $\Omega_{n}=\Omega_{n}(\lambda a, \lambda b), \Omega_{n}>0, n=1,2, \ldots$, and will assume that they are arranged in the ascending order $\Omega_{n}<\Omega_{n+1}$. In addition to the real zeros, in dependence of the values of the ratios $A_{j} / B_{j}$, equation (4.13) can have a finite set of purely imaginary solutions. The presence of such solutions leads to the modes with an imaginary frequency and, hence, to the unstable vacuum. In the consideration below we will assume the values of the coefficients in Eq. (3.2) for which the imaginary solutions are absent and the vacuum is stable. 
The coefficient $C_{\sigma}$ in formula (4.12) is determined from the normalization condition. Taking into account boundary conditions, for this coefficient one finds

$$
C_{\sigma}^{2}=\left.\frac{(2 \pi)^{1-D} \bar{I}_{i \omega}^{(a)}(\lambda a)}{\bar{I}_{i \omega}^{(b)}(\lambda b) \frac{\partial}{\partial \omega} G_{i \omega}^{a b}(\lambda a, \lambda b)}\right|_{\omega=\Omega_{n}} .
$$

Now substituting the eigenfunctions into the mode sum formula (3.3), one finds

$$
W\left(x, x^{\prime}\right)=\left.\int d \mathbf{k} \frac{e^{i \mathbf{k}\left(\mathbf{x}-\mathbf{x}^{\prime}\right)}}{(2 \pi)^{D-1}} \sum_{n=1}^{\infty} \frac{\bar{I}_{i \omega}^{(a)}(\lambda a) e^{-i \omega\left(\tau-\tau^{\prime}\right)}}{\bar{I}_{i \omega}^{(b)}(\lambda b) \frac{\partial}{\partial \omega} G_{i \omega}^{a b}(\lambda a, \lambda b)} G_{i \omega}^{(b)}(\lambda b, \lambda \xi) G_{i \omega}^{(b)}\left(\lambda b, \lambda \xi^{\prime}\right)\right|_{\omega=\Omega_{n}} .
$$

To obtain a formula for the summation over $n$, in the GAPF we choose

$$
f(z)=\frac{2 i}{\pi} F(z) \sinh \pi z, \quad g(z)=F(z) \frac{\bar{I}_{-i z}^{(a)}(\eta) \bar{I}_{i z}^{(b)}(\xi)+\bar{I}_{i z}^{(a)}(\eta) \bar{I}_{-i z}^{(b)}(\xi)}{G_{i z}^{a b}(\eta, \xi)},
$$

with a function $F(z)$ analytic in the right half-plane. The corresponding conditions are satisfied if the function $F(z)$ is restricted by the condition

$$
|F(z)|<\varepsilon(|z|) e^{-\pi x}(\xi / \eta)^{2|y|}, \quad z=x+i y, \quad x>0, \quad|z| \rightarrow \infty,
$$

where $|z| \varepsilon(|z|) \rightarrow 0$ for $|z| \rightarrow \infty$. Now from the GAPF one obtains the summation formula

$$
\begin{aligned}
\left.\sum_{n=1}^{\infty} \frac{\bar{I}_{i z}^{(a)}(\eta) \bar{I}_{-i z}^{(b)}(\xi)}{\partial G_{i z}^{a b}(\eta, \xi) / \partial z} F(z)\right|_{z=\Omega_{n}}= & \frac{1}{\pi^{2}} \int_{0}^{\infty} d x F(x) \sinh \pi x \\
& -\frac{1}{2 \pi} \int_{0}^{\infty} d z \frac{\bar{I}_{z}^{(a)}(\eta) \bar{I}_{-z}^{(b)}(\xi)}{G_{z}^{a b}(\eta, \xi)}[F(i z)+F(-i z)] .
\end{aligned}
$$

For the further evaluation of the Wightman function we apply to the sum over $n$ in Eq. (4.15) the summation formula (4.18) taking

$$
F(z)=\frac{G_{i \omega}^{(b)}(\lambda b, \lambda \xi) G_{i \omega}^{(b)}\left(\lambda b, \lambda \xi^{\prime}\right)}{\bar{I}_{i z}^{(b)}(\lambda b) \bar{I}_{-i z}^{(b)}(\lambda b)} e^{-i z\left(\tau-\tau^{\prime}\right)} .
$$

Condition (4.17) for this function is satisfied if $a^{2} e^{\left|\tau-\tau^{\prime}\right|}<\xi \xi^{\prime}$. In particular, this is the case in the coincidence limit $\tau=\tau^{\prime}$ in the region under consideration: $\xi, \xi^{\prime}>a$. As a result, for the Wightman function one obtains the expression

$$
\begin{aligned}
W\left(x, x^{\prime}\right)= & W^{(b)}\left(x, x^{\prime}\right)-\int \frac{d \mathbf{k} e^{i \mathbf{k}\left(\mathbf{x}-\mathbf{x}^{\prime}\right)}}{\pi(2 \pi)^{D-1}} \int_{0}^{\infty} d \omega \Omega_{b \omega}(\lambda a, \lambda b) \\
& \times G_{i \omega}^{(b)}(\lambda b, \lambda \xi) G_{i \omega}^{(b)}\left(\lambda b, \lambda \xi^{\prime}\right) \cosh \left[\omega\left(\tau-\tau^{\prime}\right)\right],
\end{aligned}
$$

where

$$
W^{(b)}\left(x, x^{\prime}\right)=\int \frac{d \mathbf{k} e^{i \mathbf{k}\left(\mathbf{x}-\mathbf{x}^{\prime}\right)}}{\pi^{2}(2 \pi)^{D-1}} \int_{0}^{\infty} d \omega \sinh (\pi \omega) e^{-i \omega\left(\tau-\tau^{\prime}\right)} \frac{G_{i \omega}^{(b)}(\lambda b, \lambda \xi) G_{i \omega}^{(b)}\left(\lambda b, \lambda \xi^{\prime}\right)}{\bar{I}_{i \omega}^{(b)}(\lambda b) \bar{I}_{-i \omega}^{(b)}(\lambda b)},
$$


is the Wightman function in the region $\xi<b$ for a single plate at $\xi=b$. The latter can be presented in the form

$$
W^{(b)}\left(x, x^{\prime}\right)=W_{\mathrm{R}}\left(x, x^{\prime}\right)+\left\langle\varphi(x) \varphi\left(x^{\prime}\right)\right\rangle_{\mathrm{b}},
$$

where $W_{\mathrm{R}}\left(x, x^{\prime}\right)$ is the Wightman function for the right Rindler wedge without boundaries and

$$
\left\langle\varphi(x) \varphi\left(x^{\prime}\right)\right\rangle_{\mathrm{b}}=-\int \frac{d \mathbf{k} e^{i \mathbf{k}\left(\mathbf{x}-\mathbf{x}^{\prime}\right)}}{\pi(2 \pi)^{D-1}} \int_{0}^{\infty} d \omega \frac{\bar{K}_{\omega}^{(b)}(\lambda b)}{\bar{I}_{\omega}^{(b)}(\lambda b)} I_{\omega}(\lambda \xi) I_{\omega}\left(\lambda \xi^{\prime}\right) \cosh \left[\omega\left(\tau-\tau^{\prime}\right)\right]
$$

is induced in the region $\xi<b$ by the presence of the plate at $\xi=b$. Note that the representation (4.22) with (4.23) is valid under the assumption $\xi \xi^{\prime}<b^{2} e^{\left|\tau-\tau^{\prime}\right|}$. The results of the investigation for the VEVs of the field square and the energy-momentum tensor, as well as for the vacuum interaction forces between the plates can be found in Ref. [18].

\section{Other applications}

In this section we briefly outline the problems to which the GAPF is applied in the investigation of the quantum vacuum effects. For a scalar field obeying the Robin boundary condition on two parallel plates in the Minkowski spacetime the corresponding normal modes are the zeros of the function $\left(1-b_{1} b_{2} z^{2}\right) \sin z-\left(b_{1}+b_{2}\right) z \cos z$, where the coefficients $b_{i}$ are related with the coefficients in the boundary conditions by the formula $b_{i}=B_{i} / A_{i} a$, and $a$ is the distance between the plates. The corresponding summation formula is obtained and is applied for the evaluation of the vacuum energy-momentum tensor in Ref. [19]. Similar type of series arise in the investigation of the Casimir effect for a scalar field with non-local boundary condition on parallel plates [20]. In this case the coefficients in the Robin boundary conditions are functions on the quantum numbers corresponding to the degrees of freedom parallel to the plates.

Series over the zeros of the cylindrical functions arise in the investigation of quantum vacuum effects in presence of boundaries with cylindrical symmetry. For the electromagnetic field inside a cylindrical shell the eigenmodes are zeros of the function $J_{l}(z)$ for the TM modes and the zeros of the function $J_{l}^{\prime}(z)$ for the TE modes, $l=1,2, \ldots$. The VEVs of the energy-momentum tensor in this region are evaluated in Ref. [21] making use of summation formula (3.12). For the electromagnetic vacuum in the region between two coaxial cylindrical shells with radii $a$ and $b$ the eigenmodes are zeros of the function $J_{l}(z) Y_{l}(z b / a)-Y_{l}(z) J_{l}(z b / a)$ for the TM modes and of the function $J_{l}^{\prime}(z) Y_{l}^{\prime}(z b / a)-Y_{l}^{\prime}(z) J_{l}^{\prime}(z b / a)$ for the TE modes. For the investigation of the corresponding VEVs of the energy-momentum tensor in Ref. [22] the GAPF is used. For a scalar field satisfying Robin boundary condition on cylindrically symmetric boundaries the Wightman function, VEVs for the field square and the energy-momentum tensor are investigated in Refs. [23, 24] for the cases of a single and two coaxial boundaries by using summation formulae (3.12) and (3.29). For the geometry of a wedge with the opening angle $\phi_{0}$ and with a coaxial cylindrical boundary, the eigenmodes for a Dirichlet scalar field are zeros of the function $J_{\pi l / \phi_{0}}(z)$. For the investigation of the vacuum densities in Refs. [25] the GAPF is applied to the corresponding mode-sums. The geometry of a cylindrical boundary in the cosmic string spacetime is considered in Ref. [26].

Series over the zeros of combinations of the cylindrical functions arise in the investigation of the one-loop quantum vacuum effects in the Randall-Sundrum type braneworlds. The corresponding background spacetime is described by the line element $d s^{2}=e^{-2 k_{D} y} \eta_{\mu \nu} d x^{\mu} d x^{\nu}-d y^{2}$, where 
$\eta_{\mu v}$ is the metric for the $D$-dimensional Minkowski spacetime and $k_{D}$ is the inverse radius for the background AdS spacetime. In the two-brane model the radial part of the eigenfunctions is a combination of the cylindrical functions. The corresponding eigenmodes are zeroes of the function

$$
\bar{J}_{v}^{(a)}(z) \bar{Y}_{v}^{(b)}\left(z e^{k_{D}(b-a)}\right)-\bar{Y}_{v}^{(a)}(z) \bar{J}_{v}^{(b)}\left(z e^{k_{D}(b-a)}\right), v=\sqrt{(D / 2)^{2}-D(D+1) \zeta+m^{2} / k_{D}^{2}},
$$

where $a$ and $b, a<b$, are the $y$-coordinates of the branes. The corresponding two-point function, the VEVs of the field square and the energy-momentum tensor are investigated in Ref. [27]. Similar issues for higher dimensional braneworlds with compact internal spaces are considered in [28].

\section{Conclusion}

We have considered the application of the GAPF for the renormalization of the VEVs of local physical observables in quantum field theory on manifolds with boundaries. These VEVs contain series over the eigenmodes of the corresponding problem which are zeroes of the corresponding part in the eigenfunctions. We have shown that the GAPF allows to obtain the summation formulae for the series over these zeros, which explicitly extract from the VEVs the parts corresponding to the bulk without boundaries. In addition the boundary induced parts are presented in terms of integrals which are exponentially convergent for the points away from the boundaries. As a result, the renormalization is necessary for the boundary-free parts only and this procedure is the same as that in quantum field theory without boundaries. In general, the physical quantities in the problems with boundary conditions can be classified into two main types. For the quantities of the first type the contribution of the higher modes into the boundary induced effects is suppressed by the parameters already present in the idealized model. Examples of such quantities are the local physical observables for points away from the boundaries and the interaction forces between disjoint bodies. As we have seen, the Abel-Plana formula provides an efficient way to evaluate the quantities of this type. For the quantities from the second type, such as the energy density on the boundary and the total vacuum energy, the contribution of the arbitrary higher modes is dominant and they contain divergences which cannot be eliminated by the standard renormalization procedure of quantum field theory without boundaries and additional subtractions are necessary. Methods to extract finite results for the quantities of the second type are developed in [29].

The work was supported by PVE/CAPES program and in part by the Armenian Ministry of Education and Science Grant No. 0124.

\section{References}

[1] A.A. Grib, S.G. Mamayev, V.M. Mostepanenko, Vacuum Quantum Effects in Strong Fields (Friedmann Laboratory Publishing, St. Petersburg, 1994).

[2] V.M. Mostepanenko, N.N. Trunov, The Casimir Effect and Its Applications (Oxford University Press, Oxford, 1997).

[3] M. Bordag, U. Mohidden, V.M. Mostepanenko, Phys. Rep. 353, 1 (2001); K.A. Milton, The Casimir effect: Physical Manifestation of Zero-Point Energy (World Scientific, Singapore, 2002).

[4] G. Barton, J. Phys. A 14, 1009 (1981); J. Phys. A 14, 323 (1982). 
[5] G.H. Hardy, Divergent series (Chelsea Publishing Company, New York, 1991); A. Erdélyi et al, Higher transcendental functions (McGraw Hill, New York, 1953), Vol.1; M.A. Evgrafov, Analytic functions (Nauka, Moscow, 1968, in Russian).

[6] S.G. Mamaev, V.M. Mostepanenko, A.A. Starobinsky, Sov. Phys. JETP 43, 823 (1976).

[7] A.A. Saharian, Izv. AN Arm. SSR. Matematika, 22, 166 (1987) [Sov. J. Contemp. Math. Analysis, 22, 70 (1987)].

[8] A.A. Saharian, "Electromagnetic vacuum effects in presence of macroscopic bodies," $\mathrm{PhD}$ thesis (Yerevan, 1987, in Russian).

[9] A.A. Saharian, "The generalized Abel-Plana formula. Applications to Bessel functions and Casimir effect," Preprint IC/2000/14; hep-th/0002239.

[10] A.A. Saharian, M.R. Setare, Class. Quantum Grav. 20, 3765 (2003).

[11] A.A. Saharian, Phys. Rev. D 63, 125007 (2001).

[12] L.Sh. Grigoryan, A.A. Saharian, Dokladi AN Arm. SSR 83, 28 (1986) (Reports NAS RA, in Russian).

[13] A. A. Saharian and M. R. Setare, Int. J. Mod. Phys. A 19, 4301 (2004).

[14] A.A. Saharian and E.R. Bezerra de Mello, J. Phys. A 37, 3543 (2004).

[15] E.R. Bezerra de Mello and A.A. Saharian, Class. Quantum Grav. 23, 4673 (2006).

[16] L.Sh. Grigoryan, A.A. Saharian, Izv. AN Arm. SSR. Fizika 22, 3 (1987) [Sov. J. Contemp. Phys. 22, 1 (1987)].

[17] A.A. Saharian, Class. Quantum Grav. 19, 5039 (2002).

[18] A.A. Saharian, R.M. Avagyan, R.S. Davtyan, Int. J. Mod. Phys. A 21, 2353 (2006).

[19] A. Romeo, A.A. Saharian, J. Phys. A 35, 1297 (2002).

[20] A.A. Saharian, G. Esposito, J. Phys. A 39, 5233 (2006).

[21] A.A. Saharian, Izv. AN Arm. SSR. Fizika 23, 130 (1988) [Sov. J. Contemp. Phys. 23, 14 (1988)].

[22] A.A. Saharian, Dokladi AN Arm.SSR 86, 112 (1988) (Reports NAS RA, in Russian).

[23] A. Romeo, A.A. Saharian, Phys. Rev. D 63, 105019 (2001).

[24] A.A. Saharian, A.S. Tarloyan, J. Phys. A 39, 13371 (2006).

[25] A.H. Rezaeian, A.A. Saharian, Class. Quantum Grav. 19, 3625 (2002); A.A. Saharian, A.S. Tarloyan, J. Phys. A. 38, 8763 (2005).

[26] E.R. Bezerra de Mello, V.B. Bezerra, A.A. Saharian, A.S. Tarloyan, Phys. Rev. D 74, 025017 (2006).

[27] A.A. Saharian, Nucl. Phys. B 712, 196 (2005).

[28] A.A. Saharian, Phys. Rev. D 73, 044012 (2006); A.A. Saharian, Phys. Rev. D 73, 064019 (2006).

[29] E. Elizalde, S.D. Odintsov, A. Romeo, A.A. Bytsenko, S. Zerbini, Zeta regularization techniques with applications (World Scientific, Singapore, 1994); E. Elizalde, Ten physical applications of spectral zeta functions (Springer, Berlin, 1995); E. Elizalde, S. Leseduarte, A. Romeo, J. Phys. A 26, 2409 (1993); S. Leseduarte, A. Romeo, J. Phys. A 27, 2483 (1994); M. Bordag, J. Phys. A 28, 755 (1995); M. Bordag, K. Kirsten, Phys. Rev. D 53, 5753 (1996); A.A. Bytsenko, G. Cognola, L. Vanzo, S. Zerbini, Phys. Reports 266, 1 (1996); M. Bordag, E. Elizalde, K. Kirsten, J. Math. Phys. 37, 895 (1996); S. Leseduarte, A. Romeo, Ann. Phys. (N.Y.) 250, 448 (1996); M. Bordag, K. Kirsten, J.S. Dowker, Commun. Math. Phys. 182, 371 (1996); M. Bordag, E. Elizalde, K. Kirsten, S. Leseduarte, Phys. Rev. D 56, 4896 (1997); E. Elizalde, M. Bordag, K. Kirsten, J. Phys. A31, 1743 (1998). 